\title{
Actualización del síndrome de ovario poliquístico
}

\author{
Polycystic ovary syndrome update
}

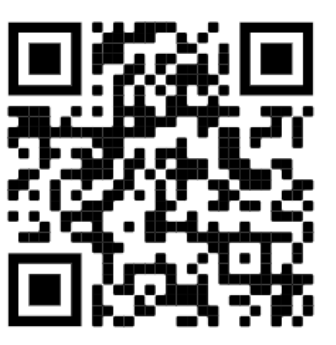

Recibido 03/11/2020

\author{
${ }^{1}$ Dra. Mary Orias Vásquez \\ Clínica de Abangares, Guanacaste, Costa Rica \\ (1D) https://orcid.org/0000-0002-2310-318X
}

Corregido $17 / 12 / 2020$
Aceptado $15 / 01 / 2021$

\section{RESUMEN}

El síndrome de ovarios poliquísticos es el más común de las endocrinopatías en mujeres. Afecta principalmente a las mujeres en edad reproductiva. Es una enfermedad de etiología desconocida, pero se cree que es multifactorial. Se manifiesta clínicamente con alteraciones menstruales, infertilidad, hirsutismo, acné, obesidad y resistencia a la insulina. Para su diagnóstico, es necesario excluir otras patologías que causen trastornos del ciclo menstrual y exceso de andrógenos, y cumplir con al menos dos de los siguientes criterios: oligomenorrea/amenorrea, hiperandrogenismo clínico y/o bioquímico, ovarios poliquísticos por ecografía. El tratamiento debe ser individualizado y enfocado en la regulación de los trastornos menstruales y en el deseo de concebir de la paciente.

PALABRAS CLAVE: anovulación; infertilidad; hiperandrogenismo; hirsutismo; síndrome de ovario poliquísticos.

\section{ABSTRACT}

Polycystic ovary syndrome is the most common endocrinopathies in women. It mainly affects women of reproductive age. It is a disease of unknown etiology, but it is believed to be multifactorial. It manifests clinically with menstrual alterations, infertility, hirsutism, acne, obesity, and insulin resistance. For its diagnosis, it is necessary to exclude other pathologies that cause disorders of the menstrual cycle and excess androgens, and meet at least two of the following criteria: oligomenorrhea / amenorrhea, clinical and / or biochemical hyperandrogenism, polycystic ovaries by ultrasound. Treatment should be individualized and focused on the regulation of menstrual disorders and the patient's desire to conceive.

KEYWORDS: anovulation; infertility; hyperandrogenism; hirsutism; polycystic ovary syndrome.

\footnotetext{
${ }^{1}$ Médica general, graduada de la Universidad de Iberoamerica (UNIBE), cod. MED15945. Correo: maye030494@hotmail.com
} 


\section{INTRODUCCIÓN}

En 1935, Stein y Leventhal describieron por primera vez el Síndrome de Ovarios Poliquísticos (SOP), en mujeres con amenorrea, hirsutismo, obesidad e histología de ovario poliquístico (1). En una conferencia del consenso de la National Institutes of health $(\mathrm{NIH})$ de los EE. UU. en 1990, se le definió como la presencia de hiperandrogenismo asociado a anovulación crónica sin otra causa especifica (2).

En el 2003, en una conferencia realizada en Roterdam, se propuso una nueva definición que incorporaba la presencia de ovarios poliquísticos en los ultrasonidos como criterio diagnóstico (3). Se propuso hacer el diagnóstico en pacientes que presentaban al menos dos de las siguientes características: hiperandrogenismo clínico o bioquímico, oligoovulación y presencia de ovarios de morfología poliquística $(2,3)$.

EI SOP presenta una incidencia entre $6 \%$ y $12 \%$, dependiendo de los criterios diagnósticos utilizados $(3,4)$. Es una de las endocrinopatías más comunes en edad reproductiva y la causa más frecuente de infertilidad oligoovulatoria.

El objetivo de esta revisión bibliográfica es analizar la información disponible sobre el SOP, con el fin de lograr señalar los datos más actuales en esta enfermedad.

\section{MÉTODO}

Para la realización de este artículo, se realizó una recopilación de información que se efectuó en las bases de datos que ofrece la Biblioteca Nacional de Salud y Seguridad Social (BINASS) Y UpToDate.

La búsqueda se realizó en las siguientes bases de datos: Pubmed, Clinical Key. Se utilizó términos como: "infertilidad", "ovario poliquístico", "criterios de Rotterdan", "cambios de estilo de vida", "anovulación".

Se obtuvieron 38 artículos tras la combinación de las diferentes palabras clave y en el buscador de internet "Google Scholar" con los mismos términos. se escogieron 16 artículos para la realización de este artículo. La búsqueda se realizó tanto en español como en inglés, se incluyeron los artículos de mayor relevancia para la realización de esta publicación.

\section{ETIOLOGÍA}

Se conoce poco sobre la etiología del SOP, pero se cree que es multifactorial, que incluye procesos neurológicos, suprarrenales, ováricos, metabólicos y ambientales, la Interrelación entre ellos aún no se logra aclarar de manera específica, por ende, persiste la incertidumbre de su origen $(5,6)$. El componente genético sigue siendo incierta, y no existe pruebas de detección genética para el diagnóstico. Tampoco se ha logrado identificar alguna sustancia ambiental especifica que contribuya a su causa (7).

\section{FISIOPATOLOGÍA}

Es compleja y se entrelaza con varios factores genéticos, metabólicos, fetales y ambientales (2-4). Destacan al menos 3 tipos de alteraciones en conjunto: disfunción neuroendocrina (hipersecreción de hormona luteinizante (LH), trastorno metabólico (resistencia a la insulina e hiperinsulinemia) y una disfunción de la esteroidogénesis y de la foliculogénesis ovárica.

- Disfunción neuroendocrina: fue la primera anomalía de laboratorio que se identificó relacionada con el SOP (1). 
Se caracteriza por un aumento de LH y una secreción de Hormona folículo estimulante $(\mathrm{FSH})$ normal o disminuida $(2,3)$. Se observa un aumento en la amplitud y frecuencia de los pulsos de $\mathrm{LH}$, lo que eleva los pulsos del factor liberador gonadotropina $(\mathrm{GnRH})$, lo cual produce un desequilibrio y aumentan los andrógenos circulantes. Esto puede deberse a una disfunción hipotalámica secundaria a los elevados niveles de insulina y andrógenos (5), porque no se ha identificado alteración en los neurotransmisores específicos para explicar esta alteración.

- Trastorno metabólico: se presenta por una resistencia periférica de insulina $(\mathrm{PI})$ que provoca una hipersecreción de esta, a su vez, promueve una mayor secreción de andrógenos por el ovario y la glándula suprarrenal, lo que estimula la secreción de LH y disminuye la síntesis de globulina trasportadora de hormona sexual (SHBG), lo que termina en un aumento de la fracción libre y actividad biológica de andrógenos (6).

La resistencia a la insulina no es factor obligatorio para establecer el diagnóstico del SOP, pero las mujeres con este síndrome tienen una elevada prevalencia a la intolerancia a la glucosa y diabetes mellitus tipo 2 (DM2) (3).

Se estima que la obesidad se presenta en $49 \%$ de las pacientes con SOP, la resistencia a la insulina se presenta en un tercio de las mujeres delgadas con SOP. Además, tienen riesgo aumentado para dislipidemia, enfermedad cardiovascular y DM2 $(3,4)$.

- Disfunción de la esteroidogénesis y foliculogénesis ovárica: se caracteriza por una alteración en la síntesis de andrógenos, lo cual está determinado por citocromo P450C17 tanto en el ovario como en la suprarrenal (6). La actividad aumentada de esta hormona produce elevación de los andrógenos ováricos y adrenales. El aumento de los andrógenos ováricos altera el desarrollo folicular y la ovulación (5).

Por otro lado, la histología del ovario poliquístico muestra folículos preantrales, de apariencia similar a los normales, pero el desarrollo más allá de este estadio no se observa, incluso puede haber detección del crecimiento y degeneraciónn (2-6). De forma característica, presenta un número aumentado de folículos, excediendo 2-3 veces a lo observado en el ovario normal.

\section{CARACTERÍSTICAS CLÍNICAS}

Este síndrome puede iniciar durante la pubertad o cerca de esta, sin embargo, el fenotipo puede ir cambiando a lo largo de la vida de la mujer haciendo que el panorama clínico sea poco predictivo. Comúnmente, se puede presentar con trastornos menstruales, infertilidad e hiperandrogenismo (7-8), pero también está vinculado a enfermedades cardiovasculares como obesidad, intolerancia a la glucosa, dislipidemia, hígado graso y apneas obstructivas del sueño (8).

- Alteraciones menstruales: se estima que se presenta entre el $75 \%$ y $85 \%$ de las mujeres con SOP, pero este porcentaje puede ser superior. Puede iniciarse con la menarca con un ligero retraso que posteriormente se acompaña de ciclos irregulares o en la prepubertad con irregularidades menstruales $(8,9)$. El patrón menstrual típico es la oligomenorrea: sangrado de menos de 
35 días o menos de 9 menstruaciones al año (9). También pueden presentarse otros patrones como amenorrea: ciclos menstruales mayores a 90 días o más de 3 meses, y polimenorrea: sangrados vaginales por menos de 25 días.

- Hiperandrogenismo: la mayoría de las mujeres tienen evidencia clínica y bioquímica de esta manifestación. Se caracteriza por acné, hirsutismo, calvicie de patrón masculino (7). En raras ocasiones se presenta con aumento de masa muscular, profundización de la voz, hipoplasia mamaria 0 clitoromegalia, estos son hallazgos de virilización y es más frecuente que se manifiesten por hipertecosis o neoplasia de ovarios y suprarrenal (3).

El hirsutismo es el exceso de vello (más grueso y pigmentado) en una distribución masculina, refleja la interacción entre los andrógenos circulantes, la concentración local y la sensibilidad del folículo piloso (10). Se puede encontrar en los pezones, labio superior, mentón y a lo largo de la línea alba del abdomen inferior $(8,9)$. Para su evaluación, se utiliza el Score Ferriman Gallwey modificado (FG) en el que se evalúan 9 regiones (labio superior, barbilla, pecho, espalda superior y baja, abdomen superior $e$ inferior, brazo, antebrazo, muslo y parte inferior de la pierna); y se otorga un puntaje del 1 al 4 en cada región, según la cantidad de vello (11).

- Ovario poliquístico: se debe realizar un ultrasonido transvaginal para apreciar el aspecto poliquístico típico de los ovarios (8). Se describe como un ovario con 120 más folículos de $2-9 \mathrm{~mm}$ o un ovario de $10 \mathrm{cc}$ por ecografía transvaginal (3-11).

El aspecto morfológico de un ovario poliquístico no es exclusivo de estas pacientes, entre el $10 \%$ y el $20 \%$ de las mujeres sanas con ciclos menstruales regulares y sin hiperandrogenismo, por lo que es importante tener presente que con el hallazgo de un ovario poliquístico por sí solo, no se establece el diagnóstico.

\section{DIAGNÓSTICO}

En el 2012, 29 expertos de diferentes países se reunieron para revisar la evidencia bibliográfica sobre este tema y acordaron el uso de criterios más amplios, es decir, los criterios de consenso de Rotterdam 2003 (Ver TABLA 1) acompañados de una descripción detallada del fenotipo de las pacientes (Ver TABLA 2) (6-10).

Desde entonces, las sociedades internacionales de EE. UU y Europa avalan el uso de esta propuesta más amplia (11).

TABLA 1. Criterios de Rotterdam

\begin{tabular}{|l|l|}
\hline Hiperandrogenismo & $\begin{array}{l}\text { Clínico o bioquímico definido como un aumento de la testosterona } \\
\text { libre o de la proteína ligadora de testosterona }\end{array}$ \\
\hline $\begin{array}{l}\text { Oligomenorrea/ } \\
\text { amenorrea }\end{array}$ & $\begin{array}{l}\text { Menos de } 8 \text { menstruaciones en } 1 \text { año o ausencia total de } \\
\text { menstruación }\end{array}$ \\
\hline Ovarios poliquísticos & $\begin{array}{l}\text { En ecografía presencia de } 12 \text { o más folículos en cada ovario con } \\
\text { una medida entre } 2 \mathrm{~mm} \text { y } 9 \mathrm{~mm} \text { o un volumen de } 10 \mathrm{ml} .\end{array}$ \\
\hline
\end{tabular}

Fuente: Síndrome de ovario poliquistico. Perez, JM, Maroto K, 2018 
TABLA 2. Diferentes fenotipos de síndrome de ovario poliquistico (según Consenso de Rotterdam)

\begin{tabular}{|l|c|c|c|}
\hline Fenotipo & Hiperandrogenismo & Oligoovulación & $\begin{array}{c}\text { Criterio } \\
\text { ecográfico }\end{array}$ \\
\hline A. Clásico & + & + & + \\
\hline B. Clásico & + & + & - \\
\hline C. Ovulatorio & + & - & + \\
\hline D. Normoandrogénico & - & + & - \\
\hline \multicolumn{2}{|l|}{ Fuente: Síndrome de ovario poliquístico. Winnykamien,I, Dalibón, A. 2017. } \\
\hline
\end{tabular}

El diagnóstico es retrospectivo y se basa en historia de oligomenorrea e hiperandrogenismo durante los años reproductivos. La poliquistosis ecográfica proporcionaría evidencia adicional, aunque es menos probable de hallar (11). Además, es importante encontrar la resistencia a la insulina, que se manifiesta como hiperglicemia, que es un hallazgo, aunque no forma parte de los criterios diagnósticos (6). También es fundamental hacer la exclusión de otras causas (hiperplasia suprarrenal congénita, tumores secretores de andrógenos y síndrome de Cushing).

\section{DIAGNÓSTICO DIFERENCIAL}

El diagnóstico de SOP se confirma cuando se han excluido otras enfermedades que comparten características con el SOP.

- Hiperandrogenismo: los andrógenos son producidos por la glándula suprarrenal como por el ovario a través del eje de retroalimentación de hormona producida por el hipotálamo y la hipófisis (12). Se deben considerar las enfermedades a nivel central que fabrican un exceso de hormona que produce una retroalimentación positiva en la producción de andrógenos, como
Prolactinoma, acromegalia y disfunción hipotalámica (3-6). También, se puede producir por cualquier patología que involucre la glándula suprarrenal encargada de la producción de dehidroepiandrosterona (DHEA) y sulfato de dehidroepiandrosterona (DHEAS). Ejemplo: Hiperplasia Suprarrenal congénita no clásica, Síndrome de Cushing, Tumores de secretores de andrógenos (3-8). Además, deben ser considerados los andrógenos producidos por el ovario, debido a que el $75 \%$ de la testosterona se produce en este órgano (12), debe ser tomado en cuenta como diagnóstico diferencial a este nivel la falla ovárica prematura.

- Amenorrea / Oligomenorrea: deben ser consideradas todas las causas de amenorrea, tomando en consideración que, en el SOP, puede estar ligado al mismo hiperandrogenismo característico. Entre las posibles causas están la falla ovárica prematura, hipotiroidismo primario, hipertiroidismo, hiperprolactinemia, hipogonadismo hipogonadotrópico, aunque en estos últimos no es característico el hiperandrogenismo (6-8). 
- Hiperinsulinemia: aunque no forma parte de los criterios diagnósticos, es un hallazgo frecuente entre este grupo de mujeres, por lo cual, es importante considerar los antecedentes personales y familiares de diabetes, así como de enfermedad cardiovascular y otras causas de hiperinsulinemia como: insulinoma, defectos genéticos de la acción de la insulina y obesidad (6-12). También, es importante descartar que sea producido por fármacos como: corticoesteroides, andrógenos, esteroides, anabolizantes, acido valproico, danazol, ciclosporina, entre otros.

\section{ABORDAJE}

- Historia clínica: se debe realizar una minuciosa historia clínica centrada en la aparición y duración de los diversos signos de exceso de andrógenos, la historia menstrual y el uso de medicamentos concomitantes, así como el uso de andrógenos exógenos. Es importante tener los antecedentes familiares de diabetes y enfermedades cardiovasculares. (Ver TABLA 3). Además, las mujeres con SOP tiene más riesgo de hiperplasia y cáncer de endometrio, infertilidad, hiperprolactinemia, trastornos del sueño y síntomas neuropsiquiátricos (7).

- Examen físico: es importante medir las cifras de tensión arterial, distribución de grasa y antropometría (IMC y circunferencia abdominal) (10).

También, se debe buscar signos de hiperandrogenismo como clitoromegalia, calvicie y la distribución de vello corporal, así como la presencia y la gravedad del acné (3).
No deben olvidarse los signos de resistencia a la insulina como hipertensión, obesidad, distribución de la grasa centrípeta y la presencia de acantosis nigricans.

La acantosis nigricans es una afectación dermatológica marcada con apariencia de musgo, aterciopelada, verrugosa e hiperpigmentada, es frecuente encontrarla en la parte posterior del cuello, axilas, debajo de los senos y vulva (7).

- Laboratorios: se debe realizar una serie de laboratorios con el fin de descartar otras patologías.

- Niveles de testosterona total- libre: se recomienda la testosterona total, pero debido a la variabilidad diaria y según el ciclo menstrual, se debe medir entre las 8-10 am durante la fase folicular (3-10). Y es el marcador más sensible, pero no se encuentra disponible para uso clínico por lo que se sugiere el índice de andrógenos libre (IAL), tiene buena correlación clínica con los niveles de testosterona libre. Su valor normal es menor a 5.

- DHEAS, androstendiona, $17 \mathrm{OH}$ progesterona, SHBG.

- FSH/LH, prolactina: la determinación de estas gonadotropinas es de poca utilidad, un cociente LH/FSH mayor a 2 , se presenta entre el $30 \%-50 \%$ de los casos de SOP (10).

\section{TRATAMIENTO}

La decisión del tratamiento debe basarse en las prioridades de la paciente, la eficacia del 
TABLA 3. Estratificación del riesgo cardiovascular en mujeres con síndrome de ovario poliquístico.

En riesgo: Mujeres con SOP con cualquiera de los siguientes factores:

Alto riesgo: Mujeres con SOP con cualquiera de los siguientes factores

- Obesidad (especialmente de grasa central)

- Fumadora

- Hipertensión

- Intolerancia a test de sobrecarga oral de glucosa.

- Dislipemia (aumento de LDL o HDL)

- Síndrome metabólico

- Diabetes Mellitus tipo 2

- Enfermedad vascular de gran vaso o afectación renal, enfermedad cardiovascular Síndrome de Apnea Obstructiva del Sueño

- Enfermedad vascular subclínica.

- Historia familiar de muerte prematura por enfermedad cardiovascular $(<55$ años en familiar varón o < 65 años en familiar mujer)

Abreviaturas: SOP: síndrome de ovario poliquístico.

Fuente: Actualización del manejo del síndrome de ovarios poliquístico. Sanchez E,2019.

tratamiento, sus efectos adversos y el deseo de quedar embarazada de la paciente.

- Cambios en el estilo de vida: es el manejo de primera línea del SOP asociado al riesgo cardiometabólico (13). El aumento del ejercicio físico y el cambio en la dieta con restricción calórica ha evidenciado una disminución del riesgo de diabetes en estas pacientes. El ejercicio 3 veces por semana durante 30 minutos ha demostrado que reduce el Índice de masa corporal, circunferencia de cintura, relación cintura- cadera y resistencia a insulina y mejora el consumo del oxígeno (13). La pérdida de peso de un 10\% mejora la función menstrual, fertilidad en paralelo con la mejoría de la resistencia a la insulina $y$ las alteraciones metabólicas (7-10).

- Screening diabetes mellitus: las mujeres con un diagnóstico de SOP deben someterse a pruebas de detección de diabetes tipo 2 e intolerancia a los carbohidratos con una prueba de glucosa en ayunas y una prueba de tolerancia de glucosa con carga de $75 \mathrm{mg}$ (7), debido a que tienen 2 a 5 veces más riesgo de desarrollarla (6). Las mujeres que se catalogan con intolerancia a los carbohidratos deben ser consideradas dentro del grupo del síndrome metabólico y buscar patologías asociadas.

- Glicemia en ayunas mayor de 126 $\mathrm{mg} / \mathrm{d}$ es DM (110- $125 \mathrm{mg} / \mathrm{dl}=$ intolerancia).

- Glucosa postcarga $75 \mathrm{~g}$ mayor de 200 $\mathrm{mg} / \mathrm{dl}$ es DM (140- $199 \mathrm{mg} / \mathrm{dl}=$ intolerancia).

- Screening de enfermedad cardiovascular: las mujeres con PCOS deben someterse a pruebas de detección de riesgo cardiovascular 
mediante la determinación del IMC, los niveles de lípidos y lipoproteínas en ayunas y los factores de riesgo del síndrome metabólico (6). Además, deben ser revisadas periódicamente por factores de riesgo de enfermedad cardiovascular porque la conversión a intolerancia a la glucosa se acerca al $20 \%$ por año. El ejercicio regular y el control de peso son métodos probados para reducir la morbilidad y mortalidad cardiovascular (13).

\section{PACIENTES SIN DESEO DE EMBARAZO}

- Anticonceptivos orales (ACO): existen varias opciones para tratar los trastornos menstruales, pero la que más se utiliza son los ACO combinados en dosis bajas como tratamiento a largo plazo (7). Además, es considerado el tratamiento de primera línea para los trastornos menstruales. Estos suprimen la secreción de gonadotropina y la producción de andrógenos ováricos y el componente estrogénico aumenta la producción hepática de globulina fijadora de hormona sexual, disminuyendo la disponibilidad de andrógenos. Confieren protección endometrial y reducen el crecimiento de vello terminal (14).

- Progestinas: los ACO de progesterona o Dispositivos Intrauterinos (DIU) que contienen progestina, son una alternativa para la protección endometrial, pero están asociadas con patrones de sangrado anormal entre el $50 \%$ y el $89 \%$ de los casos (7).

- Agentes sensibilizantes a la insulina: la metformina es una biguanida que actúa disminuyendo la producción hepática de glucosa, incrementa la sensibilidad a la insulina produciendo una mayor captación periférica (10). Se utiliza como terapia de segunda línea particularmente en mujeres con contraindicación para el uso de ACO, pero no ha demostrado que proteja el endometrio (15). Además, es una alternativa potencial para restaurar la ciclicidad menstrual en el $50 \%$ de las mujeres con SOP (16).

\section{PACIENTES CON DESEO DE EMBARAZO}

No existe un esquema para guiar las elecciones iniciales y posteriores de los métodos de inducción de la ovulación en mujeres con SOP. La sociedad estadounidense de medicina reproductiva y sociedad europea de reproducción y embriología (ASRM/ESHRE) recomiendan que se debe enfatizar en la importancia de modificadores de estilo de vida, dejar de fumar y reducir el consumo de alcohol.

- Citrato de clomifeno: anteriormente se consideraba como el tratamiento de primera línea para la inducción de la ovulación (16). La dosis es de $50 \mathrm{mg}$ a 100 mg por día por 5 días, iniciando entre el día 2 a 5 de la menstruación, la cual puede ser inducida con progesterona si fuera necesario (12). Entre un $20 \%$ y $40 \%$ tendrá un embarazo a los 6 meses de iniciado el tratamiento. Sin embargo, se ha evidenciado que suele ser menos efectivo para las tasas de nacimientos vivos que el letrozol (14).

- Letrozol: se recomienda como tratamiento de primera línea sobre el clomifeno, independiente del IMC de la paciente, principalmente en paciente 
oligoovulatoria (16). Dosis de inicio es de $2.5 \mathrm{mg}$ por 10 días, que se puede ir aumentado hasta $7.5 \mathrm{mg}$ (11). Está asociada a gestaciones gemelares (7).

- Gonadotropinas: se utiliza en mujeres que el citrato de clomifeno ha fallado. La terapia de dosis bajas con gonadotropinas ofrece más altas tasas de ovulación y desarrollo monofolicular con riesgo significativamente menor de síndrome de hiperestimulación ovárica (7).

- Perforación ovárica: se recomienda como tratamiento de segunda línea. Se realiza una perforación ovárica laparoscópica con láser o diaterna en mujeres subfértiles con anovulación y SOP indeterminado (6). Los efectos a largo plazo de la perforación ovárica laparoscópica sobre la función ovárica no están claros. No hay evidencia disponible que sugiera que mejora las tasas de nacimiento vivos cuando se utilizan solo o en combinación con citrato de clomifeno (16).

\section{CONCLUSIONES}

El SOP es una de las patologías más frecuentes que afecta la fertilidad en las mujeres en edad reproductiva. Es un trastorno complejo que involucra múltiples sistemas de órganos con inicio durante la pubertad. La lista de factores involucrados en la fisiopatología continúa expandiéndose y el papel que juega el hiperandrogenismo en los diferentes tejidos es fundamental en las características clínicas de este síndrome. Este síndrome sigue siendo una enfermedad de exclusión, por lo tanto, siempre debe tenerse presente los diagnósticos diferenciales y después de esto basarse en los criterios establecidos en el 2003 en el consenso de Rotterdam que se basa en la presencia de hiperandrogenismo clínico o bioquímico, oligomenorrea/amenorrea y la presencia de ovarios poliquísticos en la ecografía.

El tratamiento principal que se le debe recomendar a estas pacientes es la pérdida de peso mediante una dieta restrictiva, porque una disminución considerable del $10 \%$ de peso mejora la fertilidad, regula los ciclos menstruales, disminuye la resistencia a la insulina y disminuye las alteraciones metabólicas. Los ACO pueden ayudar a controlar los desórdenes menstruales en estas pacientes que no planean tener un embarazo a corto plazo. En otras palabras, el tratamiento debe ser personalizado, orientado a corregir los diversos trastornos que acompañan este síndrome, sin olvidar los deseos de las pacientes.

Debido a que implica alta demanda de salud, las estrategias están encaminadas a prevenir o por lo menos retrasar su aparición. Una de las estrategias seria identificar a las poblaciones con alto riesgo en desarrollar diabetes 2 o síndrome metabólico para una intervención oportuna y precoz.

\section{REFERENCIAS}

1. Rosenfield R, Ehrmann D. The Pathogenesis of Polycystic Ovary Syndrome (PCOS): The Hypothesis of PCOS as Functional Ovarian Hyperandrogenism Revisited. Endocrine Reviews. 2016;37(5):467-520. 
2. Dumesic D, Oberfield S, Stener-Victorin E, Marshall J, Laven J, Legro R. Scientific Statement on the Diagnostic Criteria, Epidemiology, Pathophysiology, and Molecular Genetics of Polycystic Ovary Syndrome. Endocrine Reviews [Internet]. 2015 [cited 9 August 2020];36(5):487-525. Available from: https://www.ncbi.nlm.nih.gov/pmc/articles/PMC4591526/?report=printable

3. Sanchez Gaitan E. Actualización del manejo de síndrome de ovario poliquístico. Revista Médica Sinergia [Internet]. 2019 [cited 9 August 2020];4(12):e322. Available from: https://revistamedicasinergia.com/index.php/rms

4. Mahalingaiah S, Diamanti-Kandarakis E. Targets to treat metabolic syndrome in polycystic ovary syndrome. Expert Opinion on Therapeutic Targets. 2015;19(11):1561-1574.

5. Witchel S, Oberfield S, Peña A. Polycystic Ovary Syndrome: Pathophysiology, Presentation, and Treatment With Emphasis on Adolescent Girls. Journal of the Endocrine Society. 2019;3(8):1545-1573.

6. Pérez Rojas J, Maroto Fernandez K. Síndrome de Ovarios Poliquísticos. Medicina Legal de Costa Rica Edición Virtual. 2018;35(1):1-8.

7. Practice Bulletin No. 131. Polycystic Ovary Syndrome. Obstetrics \& Gynecology. 2018;131(6):1174-1176.

8. Barbieri, MD R, Ehrmann, MD D. Diagnosing Polycystic Ovary Syndrome in Adults [Internet]. www.uptodate.com. 2018 [cited 9 August 2020]. Available from: https://www.uptodate.com/contents/diagnosis-of-polycystic-ovarysyndrome-in-adults/contributors

9. Barbieri, MD R, Ehrmann, MD D. Clinical manifestations of polycystic ovary syndrome in adults [Internet]. www.uptodate.com. 2020 [cited 9 August 2020]. Available from: https://www.uptodate.com/contents/clinicalmanifestations-of-polycystic-ovary-syndrome-in-adults/print?search=sindrome

10. Muñoz Calvo. M. Síndrome de Ovario Poliquístico (SOP). Revista de Formación Continuada de la Sociedad Española de Medicina de la Adolescencia. 2016;4(2):20-31.

11. Winnykamien I, Dalibón A, Knoblovits P. Síndrome de ovario poliquístico. Revista del Hospital de Italiano Buenos Aires. 2017;37(1):10-20.

12. Gamboa I, Prez M. Síndrome de ovario poliquístico: revisión bibliográfica. Revista médica de Costa Rica y Centroamerica. 2015; LXXII(614):175-181.

13. McCartney C, Marshall J. Polycystic Ovary Syndrome. New England Journal of Medicine. 2016;375(1):54-64.

14. De Sousa S, Norman R. Metabolic syndrome, diet and exercise. Best Practice \& Research Clinical Obstetrics \& Gynaecology [Internet]. 2016 [cited 10 August 2020];37:140-151. Available from: https://www.sciencedirect.com/science/article/abs/pii/S1521693416000079?via\%3Dihub

15. Barbieri, MD R, Ehrmann, MD D. Metformin for treatment of the polycystic ovary syndrome [Internet]. Uptodate.com. 2018 [cited 10 August 2020]. Available from: https://www.uptodate.com/contents/metformin-fortreatment-of-the-polycystic-ovary-syndrome\#!

16. Barbieri, MD R, Ehrmann, MD D. Treatment of polycystic ovary syndrome in adults [Internet]. uptodate. 2020 [cited 10 August 2020]. Available from: https://www.uptodate.com 